\title{
The Unusual Bifunctional Catalysis of Epimerization and Desaturation by Carbapenem Synthase
}

\author{
Maya Topf,, ${ }^{\S, t, \bullet}$ Gregory M. Sandala, ${ }^{\S, t, \pi}$ David M. Smith, ${ }^{t, \diamond, \star}$ Christopher J. Schofield,,,* \\ Christopher J. Easton ${ }^{\dagger, *}$ and Leo Radom ${ }^{\dagger, \pi, *}$ \\ School of Chemistry, University of Sydney, Sydney, NSW 2006, Australia, Research School of \\ Chemistry, Australian National University, Canberra, ACT 0200, Australia, and Oxford Centre for \\ Molecular Sciences and Department of Chemistry, University of Oxford, Oxford OX1 3TA, United \\ Kingdom
}

\section{SUPPORTING INFORMATION}

(Tables S1-S12, Total 12 pages including this page)

$\S$ Both authors contributed equally

$\dagger$ Australian National University

I University of Sydney

\$ Oxford Centre for Molecular Sciences

- Present address: Department of Biopharmaceutical Sciences, University of California, San Francisco, San Francisco, CA

$\checkmark$ Present address: Institute Rudjer Boskovic, Zagreb, Croatia

* Email: david.smith@irb.hr; christopher.schofield@chemistry.oxford.ac.uk; easton@ rsc.anu.edu.au;

radom@chem.usyd.edu.au 


\section{Details of Calculations}

Calculations were performed with the Gaussian $98,{ }^{1}$ GAUSSIAN 03, ${ }^{2}$ MOLPRO 2000.6, ${ }^{3}$ and ACESII $3.0^{4}$ computational packages. Unrestricted open-shell wave functions and the frozen-core approximation were employed in all cases. Geometries and scaled ${ }^{5}$ zero-point vibrational energies were obtained at the B3LYP/6-31G(d) level of theory. Improved relative energies were obtained with an improved version ${ }^{6}$ of the originally reported ${ }^{7} \mathrm{G} 3(\mathrm{MP} 2)-\mathrm{RAD}$ methodology.

(1) Frisch, M. J.; Trucks, G. W.; Schlegel, H. B.; Scuseria, G. E.; Robb, M. A.; Cheeseman, J. R.; Zakrzewski, V. G.; Montgomery, J. A., Jr.; Stratmann, R. E.; Burant, J. C.; Dapprich, S.; Millam, J. M.; Daniels, A. D.; Kudin, K. N.; Strain, M. C.; Farkas, O.; Tomasi, J.; Barone, V.; Cossi, M.; Cammi, R.; Mennucci, B.; Pomelli, C.; Adamo, C.; Clifford, S.; Ochterski, J.; Petersson, G. A.; Ayala, P. Y.; Cui, Q.; Morokuma, K.; Malick, D. K.; Rabuck, A. D.; Raghavachari, K.; Foresman, J. B.; Cioslowski, J.; Ortiz, J. V.; Stefanov, B. B.; Liu, G.; Liashenko, A.; Piskorz, P.; Komaromi, I.; Gomperts, R.; Martin, R. L.; Fox, D. J.; Keith, T.; Al-Laham, M. A.; Peng, C. Y.; Nanayakkara, A.;Challacombe, M.; Gill, P. M. W.; Johnson, B.; Chen, W.; Wong, M. W.; Andres, J. L.; Gonzalez, C.; Head-Gordon, M.; Replogle, E. S.; Pople, J. A. Gaussian 98 Revision A.11.3; Gaussian, Inc.: Pittsburgh, PA, 1998.

(2) Frisch, M. J.; Trucks, G. W.; Schlegel, H. B.; Scuseria, G. E.; Robb, M. A.; Cheeseman, J. R.; Montgomery, J. A., Jr.; T. V.; Kudin, K. N.; Burant, J. C.; Millam, J. M.; Iyengar, S. S.; Tomasi, J.; Barone, V.; Mennucci, B.; Cossi, M.; Scalmani, G.; Rega, N.; Petersson, G. A.; Nakatsuji, H.; Hada, M.; Ehara, M.; Toyota, K.; Fukuda, R.; Hasegawa, J.; Ishida, M.; Nakajima, T.; Honda, Y.; Kitao, O.; Nakai, H.; Klene, M.; Li, X.; Knox, J. E.; Hratchian, H. P.; Cross, J. B.; Adamo, C.; Jaramillo, J.; Gomperts, R.; Stratmann, R. E.; Yazyev, O.; Austin, A. J.; Cammi, R.; Pomelli, C.; Ochterski, J. W.; Ayala, P. Y.; Morokuma, K.; Voth, G. A.; Salvador, P.; Dannenberg, J. J.; Zakrzewski, G.; Dapprich, S.; Daniels, A. D.; Strain, M. C.; Farkas, O.; Malick, D. K.; Rabuck, A. D.; Raghavachari, K.; Foresman, J. B.; Ortiz, J. V.; Cui, Q.; Baboul, A. G.; Clifford, S.; Cioslowski, J.; Stefanov, B. B.; Liu, G.; Liashenko, A.; Piskorz, P.; Komaromi, I.; Martin, R. L.; Fox, D. J.; Keith, T.; Al-Laham, M. A.; Peng, C. Y.; Nanayakkara, A.; Challacombe, M.; Gill, P. M. W.; Johnson, B.; Chen, W.; Wong, M. W.; Gonzalez, C.; Pople, J. A. Gaussian 03, Revision B.03; Gaussian, Inc.: Pittsburgh, PA, 2003.

(3) Werner, H.-J.; Knowles, P. J.; Amos, R. D.; Bernhardsson, A.; Berning, A.; Celani, P.; Cooper, D. L.; Deegan, M. J. O.; Dobbyn, A. J.; Eckert, F.; Hampel, C.; Hetzer, G.; Korona, T.; Lindh, R.; Lloyd, A. W.; McNicholas, S. J.; Manby, F. R.; Meyer, W.; Mura, M. E.; Nicklass, A.; Palmieri, P.; Pitzer, R.; Rauhut, G.; Schütz, M.; Stoll, H.; Stone, A. J.; Tarroni, R.; Thorsteinsson, T. MOLPRO 2000.6; University of Birmingham: Birmingham, 1999.

(4) Stanton, J. F.; Gauss, J.; Watts, J. D.; Nooijen, M.; Oliphant, N.; Perera, S. A.; Szalay, P. G.; Lauderdale, W. J.; Kucharski, S. A.; Gwaltney, S. R.; Beck, S.; Balková, A.; Bernholdt, D. E.; Baeck, K. K.; Rozyczko, P.; Sekino, H.; Hober, C.; Bartlett, R. J. ACESII; Quantum Theory Project, University of Florida: Gainsville, 1992.

(5) Scott, A. P.; Radom, L. J. Phys. Chem. 1996, 100, 16502-16513.

(6) Henry, D. J.; Sullivan, M. B.; Radom, L. J. Chem. Phys. 2003, 118 4849-4860.

(7) Henry, D. J.; Parkinson, C. J.; Mayer, P. M.; Radom, L. J. Phys. Chem. A 2001, 105, 6750-6756. 


\section{TABLE S1: GAUSSIAN Archive Entries for the B3-LYP/6-31G(d) Optimized Geometries}

\section{$(3 S, 5 S)-1$}

$1 \backslash 1 \backslash G I N C-S C 32 \backslash F O p t \backslash R B 3 L Y P \backslash 6-31 G(d) \backslash C 7 H 9 N 103 \backslash G M S 501 \backslash 01-J u l-2003 \backslash 0 \backslash \backslash \#$ B3 LYP/6-31G(D) OPT=(CALCFC, TIGHT) MAXDISK=26214400 FREQ TEST \\3S,5S carb apenam carc-a b3lyp/6-31G* COOH consistent $\backslash \backslash 0,1 \backslash C, 0.3947738191,-1.6067$ $094524,0.8152613747 \backslash \mathrm{C}, 0.6592399082,-0.0977553255,0.5507409117 \backslash \mathrm{N},-0.431$ $1145594,0.2820105838,-0.3621846529 \backslash \mathrm{C},-1.3338679088,-0.8533828528,-0.69$ $59976799 \backslash \mathrm{C},-1.095671527,-1.8304295445,0.4652742702 \backslash \mathrm{C},-1.5015718185,1.1$ $28710725,-0.0376163939 \backslash \mathrm{C},-2.5303487354,0.1346884844,-0.6251371962 \backslash 0,-1$ $.5355645026,2.1767376403,0.5561924091 \backslash \mathrm{H},-2.9537340702,0.4356605508,-1$. $5871874745 \backslash \mathrm{H},-3.336165109,-0.1321172865,0.0633103477 \backslash \mathrm{H},-1.1302647543,-$ $1.2992151878,-1.674467853 \backslash \mathrm{H}, 0.6340651245,-1.8849211476,1.8459389174 \backslash \mathrm{H}$, $0.5946977485,0.5216048013,1.4510242894 \backslash \mathrm{H},-1.3097374158,-2.8717594042,0$ $.2063770979 \backslash \mathrm{H},-1.7318826276,-1.5598938767,1.3179600405 \backslash \mathrm{H}, 1.0367504581$, $-2.1908930463,0.1491158026 \backslash \mathrm{C}, 2.0082728088,0.1494956416,-0.124123115 \backslash 0$, $2.5701572777,-0.6197431735,-0.8718638824 \backslash 0,2.5004905503,1.3668126184,0$ $.1894761085 \backslash \mathrm{H}, 3.3284466795,1.4692977731,-0.3176287085 \backslash \backslash$ Version=DEC-AXP $-\mathrm{OSF} / 1-\mathrm{G} 03 \mathrm{RevB} .03 \backslash \mathrm{State}=1-\mathrm{A} \backslash \mathrm{HF}=-552.579353 \backslash \mathrm{RMSD}=6.955 \mathrm{e}-09 \backslash \mathrm{RMSF}=4.338 \mathrm{e}-$ $06 \backslash \mathrm{Dipole}=-0.1618894,-1.0165048,0.0478363 \backslash \mathrm{PG}=\mathrm{C} 01[\mathrm{X}(\mathrm{C} 7 \mathrm{H} 9 \mathrm{~N} 103)] \backslash \backslash @$

\section{$(3 S, 5 R)-1$}

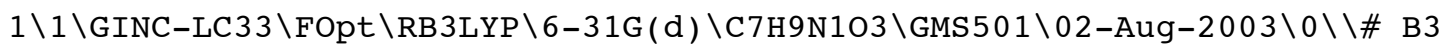
LYP $/ 6-31 \mathrm{G} *$ OPT $=($ READFC, TIGHT $)$ FREQ TEST MAXDISK $=32768000$ GEOM=CHECK GU $\mathrm{ESS}=\mathrm{READ} \backslash \backslash \mathrm{carc}-\mathrm{w}-\mathrm{b} 31 \mathrm{yp} / 6-31 \mathrm{G} * 3 \mathrm{~S}, 5 \mathrm{R}$ carbapenam $\backslash \backslash 0,1 \backslash \mathrm{C},-0.5998451138,-1$ $.2964194841,-1.4211279447 \backslash \mathrm{C}, 0.9323748522,-1.1064132454,-1.5192768357 \backslash \mathrm{C}$ $, 1.0694042052,0.4191675527,-1.4997367482 \backslash \mathrm{N},-0.0405395887,0.8450612277$, $-0.6053775526 \backslash C,-1.0818602033,-0.1796213136,-0.4401759626 \backslash \mathrm{C}, 2.06218029$ $39,1.1340622817,-0.5424448863 \backslash \mathrm{C}, 0.8318425781,1.3630336706,0.3635512628$ $\backslash 0,0.627123845,1.7670782248,1.4807242483 \backslash \mathrm{C},-1.1204508774,-0.7046123234$ $, 0.9963290539 \backslash 0,-0.2218161752,-1.3183394633,1.5272793399 \backslash 0,-2.29819361$ $27,-0.441254981,1.5984476981 \backslash \mathrm{H},-2.0699714719,0.2043959938,-0.706204464$ $2 \backslash \mathrm{H},-0.8823871393,-2.2952112263,-1.0773247565 \backslash \mathrm{H},-1.0697091149,-1.13220$ $86762,-2.3976105695 \backslash \mathrm{H}, 1.4113284336,-1.5435139905,-0.637113526 \backslash \mathrm{H}, 1.3544$ $720324,-1.5716430456,-2.4153755009 \backslash \mathrm{H}, 2.8139633632,0.4876085617,-0.0815$ $711454 \backslash \mathrm{H}, 2.5403283241,2.0397076135,-0.9249207288 \backslash \mathrm{H}, 0.9953098643,0.8551$ $876418,-2.5027005866 \backslash \mathrm{H},-2.2283440369,-0.7948045418,2.5061462205 \backslash \backslash V e r s i$ on $=x 86-$ Linux-G03RevB. 03 $\backslash$ State $=1-A \backslash H F=-552.5777726 \backslash$ RMSD $=5.389 e-09 \backslash R M S F=$ $2.925 e-06 \backslash \mathrm{Dipole}=-0.1778781,-0.7175013,-1.2230289 \backslash \mathrm{PG}=\mathrm{C} 01 \quad[\mathrm{X}(\mathrm{C} 7 \mathrm{H} 9 \mathrm{~N} 103)]$ $\backslash \backslash$ Q

\section{$(5 R)-2$}

$1 \backslash 1 \backslash$ GINC-SC55 $\backslash$ FOpt $\backslash R B 3 L Y P \backslash 6-31 G(d) \backslash C 7 H 7 N 103 \backslash G M S 501 \backslash 03-M a y-2003 \backslash 0 \backslash \backslash \#$ B3 LYP/6-31G(D) OPT=(CALCFC, TIGHT, MAXCYCLE=200) MAXDISK=104857600 FREQ TE

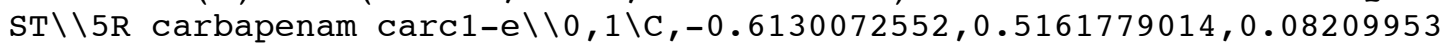
$62 \backslash \mathrm{N}, 0.4923964936,-0.2383376897,0.5768528129 \backslash \mathrm{C}, 1.6925323388,0.65607786$ $85,0.6142538515 \backslash \mathrm{C}, 1.2690837982,1.9266991254,-0.171095247 \backslash \mathrm{C},-0.22791692$ $83,1.7409961646,-0.3153257821 \backslash \mathrm{C}, 1.1870230347,-1.2364351044,-0.16868143$ $45 \backslash 0,0.8051871047,-2.2392962827,-0.7062347643 \backslash \mathrm{C}, 2.5227392321,-0.471759$ $9875,-0.0533707246 \backslash \mathrm{H}, 3.2825607966,-0.9479089089,0.5729209575 \backslash \mathrm{H}, 2.95636$ $66505,-0.2296050526,-1.027956364 \backslash \mathrm{H},-0.908862433,2.4904586939,-0.699929$ $6641 \backslash \mathrm{C},-2.0010727824,0.0050252708,0.0391833453 \backslash \mathrm{H}, 1.98918306,0.89500077$ $11,1.6394341858 \backslash \mathrm{H}, 1.5250551816,2.8420731869,0.3737984262 \backslash \mathrm{H}, 1.749195395$ 
$8,2.006920983,-1.1583606856 \backslash 0,-2.9362815027,0.6186384388,-0.434017432 \backslash$ $0,-2.1145151051,-1.2211612223,0.5908842275 \backslash \mathrm{H},-3.051686709,-1.474710748$ $6,0.4946859356 \backslash \backslash$ Version=DEC-AXP-OSF $/ 1-G 98 R e v A .11 .3 \backslash \mathrm{HF}=-551.3482762 \backslash \mathrm{RMS}$ $\mathrm{D}=3.060 \mathrm{e}-09 \backslash \mathrm{RMSF}=2.495 \mathrm{e}-06 \backslash \mathrm{Dipole}=1.0041119,0.9988028,0.3862376 \backslash \mathrm{PG}=\mathrm{C} 01$ $[\mathrm{X}(\mathrm{C} 7 \mathrm{H} 7 \mathrm{~N} 1 \mathrm{O} 3)] \backslash \backslash @$

\section{(3S,5S)-C1•}

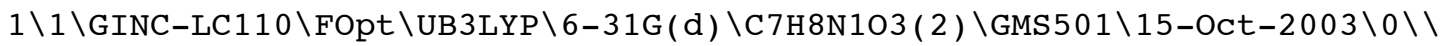
\#B3LYP/6-31G(D) OPT=TIGHT FREQ TEST MAXDISK=30146560 \\C1_5S carbapenam $\mathrm{rad} \mathrm{b} 31 \mathrm{yp} / 6-31 \mathrm{G} * \backslash \backslash 0,2 \backslash \mathrm{C}, 0.5398450442,1.7911324807,0.5719147842 \backslash \mathrm{C},-0.4$ $010964629,0.9559552462,1.3920425546 \backslash \mathrm{C},-0.6124407348,-0.3513342712,0.54$ $4218358 \backslash \mathrm{N}, 0.4875762071,-0.3248820005,-0.4201062596 \backslash \mathrm{C}, 1.0588779026,1.03$ $89217914,-0.605988578 \backslash \mathrm{C}, 1.7468117909,-0.8922028742,-0.1553720146 \backslash 0,2.0$ $492620125,-1.9669902283,0.2983848283 \backslash \mathrm{C}, 2.4899726278,0.3968540416,-0.55$ $82261258 \backslash \mathrm{C},-1.9782861999,-0.3682942076,-0.1253441508 \backslash \mathrm{H}, 3.0202395418,0$. $3526962369,-1.5137110031 \backslash \mathrm{H}, 3.1568798742,0.7762803111,0.2196637079 \backslash \mathrm{H}, 0$. $0268644255,0.6880279291,2.3714759593 \backslash \mathrm{H},-0.5413868098,-1.2576929465,1.1$ $477573861 \backslash \mathrm{H}, 0.7212996797,2.8442519659,0.7534186909 \backslash \mathrm{H},-1.3470192176,1.4$ $714687615,1.6076868653 \backslash \mathrm{H}, 0.8024510473,1.5002510224,-1.5679696287 \backslash \mathrm{O},-2$. $9077330894,-1.0624196493,0.216688347 \backslash 0,-2.0599423393,0.5308958345,-1.1$ $360592839 \backslash \mathrm{H},-2.9671584691,0.4608098258,-1.4891582575 \backslash \backslash$ Version $=x 86-\mathrm{Linu}$ $\mathrm{x}-\mathrm{G} 03$ RevB.03 $\backslash$ State $=2-\mathrm{A} \backslash \mathrm{HF}=-551.9135122 \backslash \mathrm{S} 2=0.754211 \backslash \mathrm{S} 2-1=0 . \backslash \mathrm{S} 2 \mathrm{~A}=0.75001$ $3 \backslash \mathrm{RMSD}=8.776 \mathrm{e}-09 \backslash \mathrm{RMSF}=2.592 \mathrm{e}-06 \backslash \mathrm{Dipole}=-0.0025279,1.7993264,-0.2467572$ $\backslash \mathrm{PG}=\mathrm{C} 01 \quad[\mathrm{X}(\mathrm{C} 7 \mathrm{H} 8 \mathrm{~N} 1 \mathrm{O} 3)] \backslash \backslash @$

\section{$(3 S, 5 R)-C 1 \cdot$}

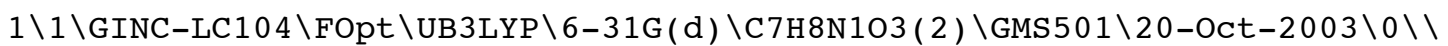
\#B3LYP/6-31G(D) OPT=(READFC) FREQ TEST MAXDISK=26214400 GUESS=READ \CC1 5R carbapenam rad chair $\mathrm{hf} / 6-31 \mathrm{G} * \backslash \backslash 0,2 \backslash \mathrm{C}, 1.5621522363,-1.6365337744,0$ $.348086 \backslash \mathrm{C}, 1.8870200629,-0.4358107275,-0.471606 \backslash \mathrm{N}, 0.5536099779,0.153318$ $0799,-0.773427 \backslash C,-0.5336358821,-0.8165770771,-0.550854 \backslash C, 0.0777992641$, $-1.8294259888,0.471912 \backslash \mathrm{C}, 2.2860038665,0.9248683301,0.199625 \backslash \mathrm{C}, 0.819000$ $8082,1.3284931183,-0.050443 \backslash 0,0.1109276742,2.256263016,0.250907 \backslash \mathrm{C},-1.8$ $303989811,-0.1307222643,-0.123868 \backslash \mathrm{H},-0.7586468106,-1.3115171095,-1.499$ $783 \backslash \mathrm{H},-0.2787167686,-1.6025780402,1.487624 \backslash \mathrm{H},-0.2399165873,-2.85814603$ $46,0.253633 \backslash \mathrm{H}, 2.5347998753,0.863395366,1.262152 \backslash \mathrm{H}, 3.0392607807,1.51888$ $64388,-0.325216 \backslash 0,-1.855078022,0.1522637321,1.197207 \backslash 0,-2.7415530173,0$ $.1195776042,-0.876365 \backslash \mathrm{H},-2.6708920959,0.6640136144,1.3537 \backslash \mathrm{H}, 2.47916709$ $64,-0.667984642,-1.368631 \backslash \mathrm{H}, 2.2976513364,-2.3298766682,0.739413 \backslash \backslash$ Versi on $=x 86-$ Linux $-G 03 R e v B .03 \backslash$ State $=2-A \backslash H F=-551.9093643 \backslash S 2=0.754222 \backslash \mathrm{S} 2-1=0 . \backslash$ $\mathrm{S} 2 \mathrm{~A}=0.750013 \backslash \mathrm{RMSD}=5.954 \mathrm{e}-09 \backslash \mathrm{RMSF}=1.256 \mathrm{e}-06 \backslash \mathrm{Dipole}=1.0028503,-1.2696939$ , $0.5615947 \backslash \mathrm{PG}=\mathrm{C} 01 \quad[\mathrm{X}(\mathrm{C} 7 \mathrm{H} 8 \mathrm{~N} 103)] \backslash \backslash @$

\section{(3S,5S)-C2•}


B3LYP/6-31G(D) FREQ TEST MAXDISK=26214400\\c2_5S carbapenam rad b31yp $/ 6-31 \mathrm{G} *$ freq $\backslash \backslash 0,2 \backslash \mathrm{C},-0.7242110247,1.875109963,-0.539601963 \backslash \mathrm{C}, 0.4629819$ $753,1.178141963,-1.140682963 \backslash C, 0.6532669753,-0.214306037,-0.577912963 \backslash$ $\mathrm{N},-0.4696990247,-0.338881037,0.345745037 \backslash \mathrm{C},-1.1420630247,0.955861963,0$ $.634719037 \backslash \mathrm{C},-1.6800290247,-0.984532037,0.051059037 \backslash 0,-1.9080120247,-2$ $.042235037,-0.479818963 \backslash \mathrm{C},-2.5058620247,0.213028963,0.573358037 \backslash \mathrm{C}, 2.02$ $88049753,-0.342158037,0.085774037 \backslash \mathrm{H},-3.0007340247,0.045941963,1.533944$ $037 \backslash \mathrm{H},-3.2309970247,0.597303963,-0.149149963 \backslash \mathrm{H},-0.8665810247,1.3741819$ $63,1.606112037 \backslash \mathrm{H}, 1.1147229753,1.601922963,-1.896031963 \backslash \mathrm{H}, 0.6031159753$, $-1.004931037,-1.337612963 \backslash \mathrm{H},-0.4906250247,2.895496963,-0.207246963 \backslash \mathrm{H},-$ 
$1.5401850247,1.974752963,-1.276201963 \backslash 0,3.0037149753,-0.806598037,-0.4$ $58200963 \backslash \mathrm{O}, 2.0509989753,0.187824963,1.330143037 \backslash \mathrm{H}, 2.9682279753,0.08868$ $1963,1.648714037 \backslash \backslash$ Version=x86-Linux-G03RevB.03 \State=2-A \HF=-551.91315 $25 \backslash S 2=0.754327 \backslash S 2-1=0 . \backslash S 2 A=0.750013 \backslash R M S D=8.630 e-09 \backslash R M S F=3.179 e-06 \backslash D i p o$ $1 e=-0.1594687,1.7361908,0.530444 \backslash \mathrm{PG}=\mathrm{C} 01[\mathrm{X}(\mathrm{C} 7 \mathrm{H} 8 \mathrm{~N} 103)] \backslash \backslash @$

\section{(3S,5R)-C2•}


\#B3LYP/6-31G(D) OPT=TIGHT FREQ TEST MAXDISK=26214400\\C2_5R carbapenam $\mathrm{rad} b 31 \mathrm{yp} / 6-31 \mathrm{G} * \backslash \backslash 0,2 \backslash \mathrm{C}, 1.2320409789,-1.7011270153,0.560076 \backslash \mathrm{C},-0.1183$ $960241,-1.9408209985,-0.052876 \backslash \mathrm{C},-0.6331220088,-0.7099149922,-0.763578$ $\backslash \mathrm{N}, 0.5764620015,0.1231789929,-0.826123 \backslash \mathrm{C}, 1.791528993,-0.5641750222,-0$. $318366 \backslash \mathrm{C},-1.7865060004,-0.0337259779,-0.000982 \backslash 0,-2.8728879975,0.20207$ $90356,-0.470714 \backslash C, 0.8779280164,1.3266479891,-0.174004 \backslash 0,0.244825029,2$. $343015997,-0.036269 \backslash \mathrm{C}, 2.2511400098,0.7883459721,0.290521 \backslash 0,-1.45485599$ $74,0.212523018,1.287605 \backslash \mathrm{H}, 3.1226130156,1.2590619613,-0.172005 \backslash \mathrm{H}, 2.3644$ $790099,0.7963339707,1.37824 \backslash \mathrm{H},-1.0322460115,-0.9284269872,-1.761631 \backslash \mathrm{H}$, $2.4260769882,-0.9509840301,-1.122805 \backslash \mathrm{H},-2.2063689914,0.6955280273,1.67$ $9681 \backslash \mathrm{H},-0.7345040349,-2.8160189909,0.119645 \backslash \mathrm{H}, 1.135989983,-1.373468014$ $1,1.610211 \backslash \mathrm{H}, 1.8643909678,-2.5966080231,0.561805 \backslash \backslash$ Version=x86-Linux-G0 3RevB.03\State $=2-\mathrm{A} \backslash \mathrm{HF}=-551.9104713 \backslash \mathrm{S} 2=0.754244 \backslash \mathrm{S} 2-1=0 . \backslash \mathrm{S} 2 \mathrm{~A}=0.750013 \backslash \mathrm{RM}$ $\mathrm{SD}=7.480 \mathrm{e}-09 \backslash \mathrm{RMSF}=1.869 \mathrm{e}-06 \backslash \mathrm{Dipole}=1.0100621,-1.3638566,0.6582999 \backslash \mathrm{PG}=\mathrm{C}$ $01[\mathrm{X}(\mathrm{C} 7 \mathrm{H} 8 \mathrm{~N} 103)] \backslash \backslash @$

\section{(5S)-C3.}


B3LYP/6-31G(D) OPT=(TIGHT, MAXCYCLE=200) FREQ TEST MAXDISK=65536000\\3 $\mathrm{S}, 5 \mathrm{~S}$ carbapenam3-carboxylate (c3 radical) carc1-h1 OH in $\backslash \backslash 0,2 \backslash \mathrm{C},-1.189$ $6236209,1.9214536409,-0.3433679111 \backslash \mathrm{C}, 0.3432365528,1.9311982606,-0.0776$ $381676 \backslash \mathrm{C}, 0.6684696625,0.4742491074,0.1558251363 \backslash \mathrm{N},-0.5022202216,-0.188$ $5221353,0.4777802301 \backslash \mathrm{C},-1.6674937996,0.7311671752,0.5047592392 \backslash \mathrm{C},-1.25$ $76085592,-1.2624681688,-0.0968565647 \backslash 0,-0.9410704527,-2.3547609285,-0$. $4735112765 \backslash \mathrm{C},-2.547345906,-0.4146663901,-0.0613596908 \backslash \mathrm{H},-3.3388482864$, $-0.7948690733,0.5903290274 \backslash \mathrm{H},-2.9543413343,-0.2359876907,-1.0610842757$ $\backslash \mathrm{H},-1.9223835536,1.0314548788,1.5272395573 \backslash \mathrm{H}, 0.9312877301,2.3399069363$ $,-0.9048008688 \backslash \mathrm{C}, 1.9770284025,-0.1079653496,0.0277074737 \backslash \mathrm{H},-1.67652501$ $44,2.8631732711,-0.0762870228 \backslash \mathrm{H},-1.380203782,1.7287056143,-1.406118686$ $3 \backslash \mathrm{H}, 0.5850733249,2.538321576,0.8079407357 \backslash \mathrm{O}, 2.9666654951,0.526434715,-$ $0.3243186364 \backslash 0,2.0202931442,-1.4322187827,0.3392030952 \backslash \mathrm{H}, 2.944400582$, $1.7045002499,0.1929173743 \backslash \backslash$ Version=DEC-AXP-OSF / 1-G98RevA. $11.3 \backslash \mathrm{HF}=-551$. $9390021 \backslash S 2=0.755888 \backslash S 2-1=0 . \backslash S 2 A=0.750025 \backslash R M S D=7.069 e-09 \backslash R M S F=4.355 e-06$ $\backslash$ Dipole $=-1.3106894,1.020269,0.2773379 \backslash \mathrm{PG}=\mathrm{C} 01[\mathrm{X}(\mathrm{C} 7 \mathrm{H} 8 \mathrm{~N} 103)] \backslash \backslash @$

\section{TS:(5S)-C3• $\rightarrow(5 S)-3$}

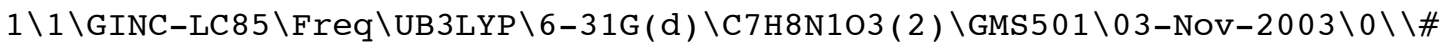
B3LYP/6-31G* FREQ TEST MAXDISK=26214400\\3S,5S carbapenam ts between C3 and c5 radical ( $3=\mathrm{N})$ carc-hr-ts2 freq $\backslash \backslash 0,2 \backslash \mathrm{C},-0.1300839012,1.17103$ $09753,1.0159341111 \backslash \mathrm{C}, 0.7121530988,1.9253659753,-0.0831598889 \backslash \mathrm{C}, 1.37032$ $20988,0.9309119753,-1.0101718889 \backslash \mathrm{C}, 2.4938230988,0.0458669753,-0.527009$ $8889 \backslash \mathrm{C}, 1.5912340988,-0.9651230247,0.1670201111 \backslash 0,1.8312870988,-1.79551$ $10247,1.0069681111 \backslash \mathrm{C},-0.6251659012,-0.0516530247,0.2933601111 \backslash \mathrm{N}, 0.3044$ $390988,-0.7159880247,-0.3897648889 \backslash \mathrm{H}, 3.1925440988,0.5153809753,0.17504$ $01111 \backslash \mathrm{H}, 3.0712770988,-0.4055540247,-1.3401978889 \backslash \mathrm{H}, 1.2051970988,1.0359$ $379753,-2.0778518889 \backslash \mathrm{H}, 0.0439280988,2.5739679753,-0.6577428889 \backslash \mathrm{H}, 1.461$ $3190988,2.5695079753,0.3955641111 \backslash \mathrm{H},-0.9390219012,1.7905929753,1.40569$ 
$81111 \backslash \mathrm{H}, 0.5191090988,0.8821899753,1.8513881111 \backslash \mathrm{C},-2.0619939012,-0.1932$ $930247,-0.0188108889 \backslash 0,-2.3448349012,-1.2434870247,-0.8217538889 \backslash 0,-2$. $9206279012,0.5520469753,0.4253921111 \backslash \mathrm{H},-3.3137529012,-1.2331400247,-0$. 9313648889 \\Version=x86-Linux-G03RevB.03 \State=2-A \HF=-551.8903562 \S2= $0.787101 \backslash \mathrm{S} 2-1=0 . \backslash \mathrm{S} 2 \mathrm{~A}=0.750319 \backslash \mathrm{RMSD}=6.181 \mathrm{e}-09 \backslash \mathrm{RMSF}=2.303 e-06 \backslash \mathrm{Dipole}=0.5$ $536343,1.2145208,-0.7739613 \backslash \mathrm{PG}=\mathrm{C} 01[\mathrm{X}(\mathrm{C} 7 \mathrm{H} 8 \mathrm{~N} 103)] \backslash \backslash @$

\section{(5S)-3}

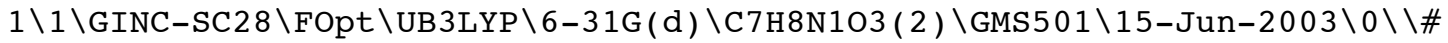
B3LYP/6-31G* OPT $=($ TIGHT, CALCFC, MAXCYCLE=200) FREQ MAXDISK=26214400 TE $\mathrm{ST} \backslash \backslash 3 \mathrm{~S}, 5 \mathrm{~S}$ carbapenam -7 membered ring (c5 radical, C3=N) carc1-rti tight $\backslash \backslash 0,2 \backslash \mathrm{C},-0.0778013584,1.0306192538,1.0492961355 \backslash \mathrm{C}, 0.6514107582,2.00655$ $23114,0.08610966 \backslash \mathrm{C}, 1.5902943569,1.3526127869,-0.884378785 \backslash \mathrm{C}, 2.31547248$ $14,0.0650048316,-0.6328018755 \backslash \mathrm{C}, 1.5242089589,-1.0412938326,0.075943085$ $1 \backslash 0,2.0476737488,-1.8821422084,0.7748051889 \backslash \mathrm{C},-0.6029294748,-0.1542578$ $431,0.2710808474 \backslash \mathrm{N}, 0.1372260226,-1.082255003,-0.1964307114 \backslash \mathrm{H}, 3.2242755$ $834,0.2005891814,-0.0218853903 \backslash \mathrm{H}, 2.6680295087,-0.3530144528,-1.5866597$ $013 \backslash \mathrm{H}, 1.8997881165,1.9382226498,-1.7459139425 \backslash \mathrm{H},-0.1019664848,2.574790$ $1126,-0.4761869872 \backslash \mathrm{H}, 1.1780221927,2.7555771952,0.7047196241 \backslash \mathrm{H},-0.89092$ $19986,1.5530728521,1.5558611019 \backslash \mathrm{H}, 0.6184797808,0.6643099769,1.81246906$ $7 \backslash \mathrm{C},-2.0798548497,-0.1624592004,-0.0550533872 \backslash 0,-2.8144430481,0.765768$ $1301,0.216020466 \backslash 0,-2.4880368909,-1.2805102986,-0.6766701909 \backslash \mathrm{H},-3.4426$ $445706,-1.1633573236,-0.841806586 \backslash \backslash$ Version=DEC-AXP-OSF / 1-G03RevB . 03\St ate $=2-\mathrm{A} \backslash \mathrm{HF}=-551.9179453 \backslash \mathrm{S} 2=0.754042 \backslash \mathrm{S} 2-1=0 . \backslash \mathrm{S} 2 \mathrm{~A}=0.750011 \backslash \mathrm{RMSD}=9.742 \mathrm{e}-0$ $9 \backslash \mathrm{RMSF}=3.951 \mathrm{e}-06 \backslash \mathrm{Dipole}=-0.1562959,1.0805662,-0.4573129 \backslash \mathrm{PG}=\mathrm{C} 01] \mathrm{X}(\mathrm{C} 7 \mathrm{H} 8$ $\mathrm{N} 103$ ) $] \backslash \backslash[$

\section{TS:(5S)-3 $\rightarrow(5 R)-3$}

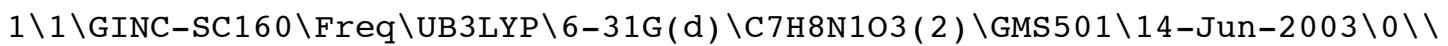
\#N GEOM=ALLCHECK GUESS=READ SCRF=CHECK TEST GENCHK UB3LYP/6-31G(D) FRE $\mathrm{Q} \backslash \backslash$ carbapenam $\mathrm{TS} \mathrm{b} / \mathrm{w} 3 \mathrm{~S}, 5 \mathrm{~S}$ and $3 \mathrm{~S}, 5 \mathrm{R}$ of $\mathrm{c} 5$ radical carc-r2r1-ts 1 b3lyp $/ 6-31 \mathrm{G} * \backslash \backslash 0,2 \backslash \mathrm{C},-0.5590685281,-2.0463377885,-1.0869125535 \backslash \mathrm{C}, 0.922527080$ $2,-2.0880317758,-0.9150954672 \backslash \mathrm{C}, 1.7525039135,-0.8570526921,-1.06509820$ $72 \backslash \mathrm{C}, 0.9056593161,0.4302823733,-1.1687491182 \backslash \mathrm{C}, 0.0326106608,0.66272979$ $27,0.048774043 \backslash \mathrm{N},-0.849062938,-0.0866839104,0.5979374507 \backslash \mathrm{C},-1.25298450$ $16,-1.3741287156,0.1165210097 \backslash 0,-2.1495680054,-1.9361762496,0.70556286$ $21 \backslash \mathrm{C}, 0.2775087137,2.0028201709,0.7403084636 \backslash 0,1.1402031824,2.782289051$ $9,0.3866136665 \backslash \mathrm{O},-0.5470132266,2.2357425034,1.7720379941 \backslash \mathrm{H},-0.84722875$ $22,-1.4934627972,-1.9947615389 \backslash \mathrm{H},-0.9906929081,-3.0464976604,-1.163416$ $8168 \backslash \mathrm{H}, 1.3809754902,-2.9977372931,-0.5390483027 \backslash \mathrm{H}, 2.3862021762,-0.9136$ $70809,-1.9668219594 \backslash \mathrm{H}, 2.4585431325,-0.7645406157,-0.2256669426 \backslash \mathrm{H}, 0.268$ $0084989,0.3803255837,-2.0603878018 \backslash \mathrm{H}, 1.5554925414,1.2975809192,-1.2925$ $73406 \backslash \mathrm{H},-0.2893751438,3.1082594091,2.1249094108 \backslash \backslash$ Version=DEC-AXP-OSF / 1 $-\mathrm{G} 03$ RevB.03 $\backslash$ State $=2-\mathrm{A} \backslash \mathrm{HF}=-551.9105022 \backslash \mathrm{S} 2=0.755539 \backslash \mathrm{S} 2-1=0 . \backslash \mathrm{S} 2 \mathrm{~A}=0.750023$ $\backslash \mathrm{RMSD}=4.772 \mathrm{e}-09 \backslash \mathrm{RMSF}=2.173 \mathrm{e}-06 \backslash \mathrm{Dipole}=0.9629716,0.1950333,-0.8486609 \backslash$ $\mathrm{PG}=\mathrm{C} 01[\mathrm{X}(\mathrm{C} 7 \mathrm{H} 8 \mathrm{~N} 103)] \backslash \backslash @$

\section{$(5 R)-3$}


N GEOM=ALLCHECK GUESS=READ SCRF=CHECK TEST GENCHK UB3LYP/6-31G(D) FREQ

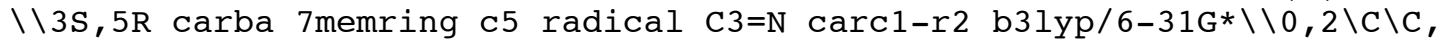
$1, \mathrm{~B} 1 \backslash \mathrm{C}, 2, \mathrm{~B} 2,1, \mathrm{~A} 1 \backslash \mathrm{C}, 3, \mathrm{~B} 3,2, \mathrm{~A} 2,1, \mathrm{D} 1,0 \backslash \mathrm{C}, 4, \mathrm{~B} 4,3, \mathrm{~A} 3,2, \mathrm{D} 2,0 \backslash \mathrm{N}, 5, \mathrm{~B} 5,4, \mathrm{~A} 4,3, \mathrm{D}$ $3,0 \backslash \mathrm{C}, 6, \mathrm{~B} 6,5, \mathrm{~A} 5,4, \mathrm{D} 4,0 \backslash 0,7, \mathrm{~B} 7,6, \mathrm{~A} 6,5, \mathrm{D} 5,0 \backslash \mathrm{C}, 5, \mathrm{~B} 8,4, \mathrm{~A} 7,6, \mathrm{D} 6,0 \backslash 0,9, \mathrm{~B} 9,5$, $\mathrm{A} 8,4, \mathrm{D} 7,0 \backslash 0,9, \mathrm{~B} 10,5, \mathrm{~A} 9,10, \mathrm{D} 8,0 \backslash \mathrm{H}, 1, \mathrm{~B} 11,2, \mathrm{~A} 10,3, \mathrm{D} 9,0 \backslash \mathrm{H}, 1, \mathrm{~B} 12,2, \mathrm{~A} 11,12, \mathrm{D}$ $10,0 \backslash \mathrm{H}, 2, \mathrm{~B} 13,1, \mathrm{~A} 12,3, \mathrm{D} 11,0 \backslash \mathrm{H}, 3, \mathrm{~B} 14,2, \mathrm{~A} 13,4, \mathrm{D} 12,0 \backslash \mathrm{H}, 3, \mathrm{~B} 15,2, \mathrm{~A} 14,4, \mathrm{D} 13,0$ $\backslash \mathrm{H}, 4, \mathrm{~B} 16,3, \mathrm{~A} 15,5, \mathrm{D} 14,0 \backslash \mathrm{H}, 4, \mathrm{~B} 17,3, \mathrm{~A} 16,5, \mathrm{D} 15,0 \backslash \mathrm{H}, 11, \mathrm{~B} 18,9, \mathrm{~A} 17,5, \mathrm{D} 16,0 \backslash \backslash \mathrm{B}$ 
$1=1.49903462 \backslash \mathrm{B} 2=1.50035751 \backslash \mathrm{B} 3=1.55315866 \backslash \mathrm{B} 4=1.51178143 \backslash \mathrm{B} 5=1.27576031 \backslash \mathrm{B}$ $6=1.41396783 \backslash B 7=1.21222303 \backslash B 8=1.51254155 \backslash B 9=1.21436579 \backslash B 10=1.34280826 \backslash$ $\mathrm{B} 11=1.10343129 \backslash \mathrm{B} 12=1.09949731 \backslash \mathrm{B} 13=1.08671672 \backslash \mathrm{B} 14=1.10499819 \backslash \mathrm{B} 15=1.0984$ $8046 \backslash \mathrm{B} 16=1.09610678 \backslash \mathrm{B} 17=1.0912102 \backslash \mathrm{B} 18=0.97586171 \backslash \mathrm{A} 1=124.63493098 \backslash \mathrm{A} 2=11$ $4.91464513 \backslash A 3=109.70260212 \backslash A 4=123.86984465 \backslash A 5=118.516851 \backslash A 6=120.968055$ $83 \backslash A 7=117.04947263 \backslash A 8=122.58492451 \backslash A 9=113.63871046 \backslash A 10=112.67406055 \backslash A 1$ $1=109.63768627 \backslash A 12=117.27525369 \backslash A 13=111.06944306 \backslash A 14=108.86859226 \backslash A 15=$ $110.08473322 \backslash \mathrm{A} 16=109.68824599 \backslash \mathrm{A} 17=106.01085305 \backslash \mathrm{D} 1=28.49394567 \backslash \mathrm{D} 2=45.36$ $986687 \backslash D 3=-72.85774576 \backslash D 4=-2.97273798 \backslash D 5=-113.31920433 \backslash D 6=175.03797433$ $\backslash D 7=-4.19889568 \backslash D 8=180.51337373 \backslash D 9=81.28807802 \backslash D 10=116.84764728 \backslash D 11=19$ $1.4764282 \backslash D 12=-122.43228144 \backslash D 13=122.15062017 \backslash D 14=-119.29346478 \backslash D 15=121$ $.92864085 \backslash D 16=179.35717961 \backslash \backslash$ Version=DEC-AXP-OSF / 1-G03RevB . 03 \State=2-A $\backslash \mathrm{HF}=-551.9179447 \backslash \mathrm{S} 2=0.754042 \backslash \mathrm{S} 2-1=0 . \backslash \mathrm{S} 2 \mathrm{~A}=0.750011 \backslash \mathrm{RMSD}=5.459 \mathrm{e}-09 \backslash \mathrm{RMSF}=$ $2.805 e-05 \backslash \mathrm{Dipole}=-0.4162164,-0.2469548,1.0804823 \backslash \mathrm{PG}=\mathrm{C} 01 \quad[\mathrm{X}(\mathrm{C} 7 \mathrm{H} 8 \mathrm{~N} 103)]$ $\backslash \backslash @$

\section{$\mathrm{TS}:(5 R)-3 \rightarrow(5 R)-\mathrm{C} 3 \cdot$}

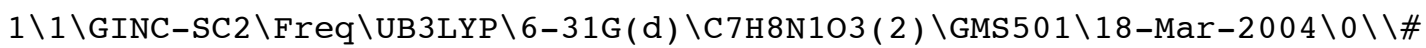
B3LYP/6-31G* MAXDISK=45875200 FREQ=NORAMAN \\3S,5R carbapenam TS enanti omer of $5 \mathrm{~S}$ b3lyp/6-31G* freq from Molecule $\backslash \backslash 0,2 \backslash C, 0.1300790617,1.17102$ $88272,1.0159293827 \backslash \mathrm{C},-0.7121509383,1.9253688272,-0.0831606173 \backslash \mathrm{C},-1.370$ $3209383,0.9309088272,-1.0101706173 \backslash \mathrm{C},-2.4938209383,0.0458678272,-0.527$ $0106173 \backslash \mathrm{C},-1.5912309383,-0.9651211728,0.1670193827 \backslash 0,-1.8312899383,-1$. $7955111728,1.0069693827 \backslash \mathrm{C}, 0.6251690617,-0.0516511728,0.2933593827 \backslash \mathrm{N},-0$ $.3044409383,-0.7159911728,-0.3897606173 \backslash \mathrm{H},-3.1925409383,0.5153778272,0$ $.1750393827 \backslash \mathrm{H},-3.0712809383,-0.4055521728,-1.3402006173 \backslash \mathrm{H},-1.205200938$ $3,1.0359388272,-2.0778506173 \backslash \mathrm{H},-0.0439319383,2.5739688272,-0.657740617$ $3 \backslash \mathrm{H},-1.4613219383,2.5695088272,0.3955593827 \backslash \mathrm{H}, 0.9390190617,1.790588827$ $2,1.4056993827 \backslash \mathrm{H},-0.5191109383,0.8821888272,1.8513893827 \backslash \mathrm{C}, 2.061989061$ $7,-0.1932901728,-0.0188106173 \backslash 0,2.3448390617,-1.2434901728,-0.82175061$ $73 \backslash 0,2.9206290617,0.5520498272,0.4253893827 \backslash \mathrm{H}, 3.3137490617,-1.23314017$ $28,-0.9313706173 \backslash \backslash$ Version=DEC-AXP-OSF $/ 1-G 03$ RevB . 03 $\backslash$ State $=2-A \backslash H F=-551.8$ $903562 \backslash \mathrm{S} 2=0.787101 \backslash \mathrm{S} 2-1=0 . \backslash \mathrm{S} 2 \mathrm{~A}=0.750319 \backslash \mathrm{RMSD}=6.181 \mathrm{e}-09 \backslash \mathrm{RMSF}=3.894 \mathrm{e}-06 \backslash$ Dipole $=-0.5536487,1.2145502,-0.7739735 \backslash \mathrm{PG}=\mathrm{C} 01[\mathrm{X}(\mathrm{C} 7 \mathrm{H} 8 \mathrm{~N} 103)] \backslash \backslash @$

\section{(5R)-C3•}

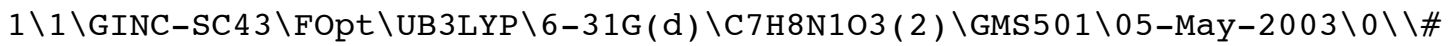
B3LYP/6-31G* OPT=TIGHT TEST MAXDISK=65536000 \\3S,5R carbapenam-3-carb oxylate ( $\mathrm{C} 3$ radical) $\mathrm{OH}$ in to $\mathrm{N} \backslash \backslash 0,2 \backslash \mathrm{C},-0.7512174494,0.3548337071,-1.7$ $785210415 \backslash \mathrm{C},-0.7085999321,0.3493071035,-0.2681778998 \backslash \mathrm{N}, 0.6156813642,0$. $3459794602,0.1314533876 \backslash \mathrm{C}, 1.5421877548,0.3655896675,-1.0285561439 \backslash \mathrm{C}, 0$. $6646417532,-0.1480181756,-2.1820637442 \backslash \mathrm{C}, 1.450361393,-0.539101092,0.88$ $91590941 \backslash \mathrm{C}, 2.51283366,-0.545004829,-0.2309168779 \backslash 0,1.302660055,-1.0656$ $813265,1.9550233818 \backslash \mathrm{H}, 3.4804582387,-0.1139435947,0.0404080115 \backslash \mathrm{H}, 2.6651$ $420326,-1.5426708404,-0.6533919359 \backslash \mathrm{H},-1.5651952036,-0.2728545544,-2.15$ $33437096 \backslash \mathrm{H},-0.933943736,1.3685082782,-2.1663478546 \backslash \mathrm{C},-1.8635755866,0.3$ $201611119,0.5879158548 \backslash \mathrm{H}, 1.9267875655,1.3731483203,-1.2222469746 \backslash \mathrm{H}, 0.9$ $852648411,0.216115444,-3.1617824469 \backslash \mathrm{H}, 0.6755896481,-1.2444816751,-2.20$ $57291095 \backslash 0,-1.5635479019,0.3711240016,1.9145411889 \backslash 0,-3.0146176014,0.2$ $600820406,0.1668933861 \backslash \mathrm{H},-2.4196189061,0.3235197139,2.377561203 \backslash \backslash$ Versi on=DEC-AXP-OSF $/ 1-G 98 R e v A .11 .3 \backslash \mathrm{HF}=-551.9390021 \backslash \mathrm{S} 2=0.755888 \backslash \mathrm{S} 2-1=0 . \backslash \mathrm{S} 2 \mathrm{~A}=$ $0.750025 \backslash \mathrm{RMSD}=2.231 \mathrm{e}-09 \backslash \mathrm{RMSF}=4.365 \mathrm{e}-06 \backslash \mathrm{Dipole}=1.0944001,0.2583811,-1.2$ $535089 \backslash \mathrm{PG}=\mathrm{C} 01 \quad[\mathrm{X}(\mathrm{C} 7 \mathrm{H} 8 \mathrm{~N} 103)] \backslash \backslash @$ 


\section{$(3 S, 5 S)-C 5 \cdot$}

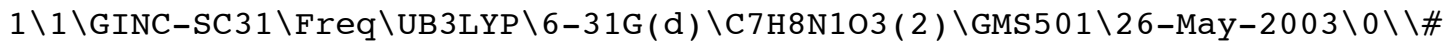
B3LYP/6-31G* FREQ TEST MAXDISK=39321600\\3S,5S carbapenam-3-carboxylat

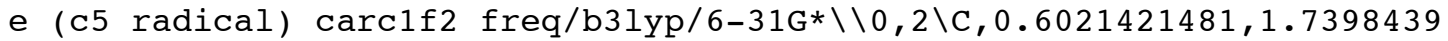
$877,-0.2673031235 \backslash \mathrm{C}, 0.6619021481,0.1822119877,-0.4407391235 \backslash \mathrm{N},-0.56355$ $08519,-0.2339750123,0.2207008765 \backslash \mathrm{C},-1.4347558519,0.8357469877,0.569088$ $8765 \backslash C,-0.9063008519,2.0775199877,-0.0985611235 \backslash C,-1.6139468519,-1.104$ $9790123,-0.1338751235 \backslash \mathrm{C},-2.6602968519,-0.0506650123,0.3309458765 \backslash 0,-1$. $6147008519,-2.1886720123,-0.6574881235 \backslash \mathrm{C}, 1.9210071481,-0.4023300123,0$. $1842528765 \backslash \mathrm{H},-3.2602488519,-0.3238720123,1.2035608765 \backslash \mathrm{H},-3.3244528519$, $0.2642539877,-0.4825661235 \backslash \mathrm{H}, 1.0703431481,2.2540919877,-1.1098951235 \backslash \mathrm{H}$ $, 0.6367841481,-0.1003270123,-1.4999521235 \backslash \mathrm{H},-1.0566398519,2.9985089877$ $, 0.4743368765 \backslash \mathrm{H},-1.3804648519,2.2282939877,-1.0840271235 \backslash \mathrm{H}, 1.143148148$ $1,2.0187249877,0.6429468765 \backslash 0,2.9623061481,-0.2853800123,-0.6777521235$ $\backslash 0,2.0216401481,-0.8668350123,1.2927498765 \backslash \mathrm{H}, 3.7439171481,-0.638847012$ $3,-0.2122421235 \backslash \backslash$ Version=DEC-AXP-OSF / 1-G03RevB. 02 \State=2-A $\backslash H F=-551.91$ $27419 \backslash \mathrm{S} 2=0.753074 \backslash \mathrm{S} 2-1=0 . \backslash \mathrm{S} 2 \mathrm{~A}=0.750007 \backslash \mathrm{RMSD}=3.314 \mathrm{e}-09 \backslash \mathrm{RMSF}=2.690 \mathrm{e}-06 \backslash \mathrm{D}$ ipole $=0.2826805,1.5623596,-0.4908171 \backslash \mathrm{PG}=\mathrm{C} 01 \quad[\mathrm{X}(\mathrm{C} 7 \mathrm{H} 8 \mathrm{~N} 1 \mathrm{O} 3)] \backslash \backslash @$

\section{$\mathrm{TS}:(3 S, 5 S)-\mathrm{C} 5 \bullet \rightarrow(3 S, 5 R)-\mathrm{C} 5 \bullet$}

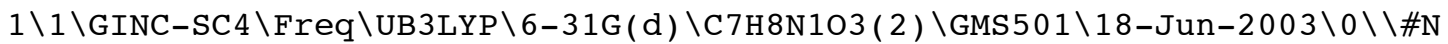
GEOM=ALLCHECK GUESS=READ SCRF=CHECK TEST GENCHK UB3LYP/6-31G(D) FREQ \ \carbapenam $\mathrm{TS} \mathrm{b} / \mathrm{w} \mathrm{C} 5 \mathrm{rad}$ of $3 \mathrm{~S}, 5 \mathrm{~S}$ and $3 \mathrm{~S}, 5 \mathrm{R} \mathrm{b} 31 \mathrm{yp} / 6-31 \mathrm{G} * \backslash \backslash 0,2 \backslash \mathrm{C},-0.95$ $80568114,0.0788475546,0.4196122483 \backslash \mathrm{N}, 0.4993154491,0.0343980649,0.43397$ $74451 \backslash \mathrm{C}, 1.1205337881,1.2839591702,0.4395577789 \backslash \mathrm{C}, 0.1118409358,2.372634$ $0084,0.5034739939 \backslash \mathrm{C},-1.1970491729,1.596474697,0.1164658719 \backslash \mathrm{C}, 1.6816252$ $135,-0.7064885718,0.322146357 \backslash 0,1.9184225972,-1.8863232713,0.243296999$ $8 \backslash \mathrm{C}, 2.4929263899,0.6465796462,0.3466896177 \backslash \mathrm{C},-1.5256275528,-0.83104851$ $49,-0.67092074 \backslash \mathrm{H}, 3.1563780267,0.7438427291,1.2131742974 \backslash \mathrm{H}, 3.0638126217$ $, 0.8267810857,-0.5709411822 \backslash \mathrm{H},-1.3716171385,1.6947941456,-0.9580729208$ $\backslash \mathrm{H},-2.0776008504,1.9711649675,0.6455090179 \backslash \mathrm{H},-1.3620177685,-0.22833483$ $78,1.3898516338 \backslash \mathrm{H}, 0.0171040222,2.8203205378,1.5080373302 \backslash \mathrm{H}, 0.293381947$ $6,3.2036879969,-0.1898978776 \backslash 0,-2.0948859395,-1.9339449692,-0.14070712$ $34 \backslash 0,-1.470804616,-0.6093522419,-1.8583375071 \backslash \mathrm{H},-2.3936620781,-2.48182$ $71584,-0.8916721348 \backslash \backslash$ Version=DEC-AXP-OSF $/ 1-G 03$ RevB . 03 \State $=2-A \backslash H F=-55$ $1.9055675 \backslash \mathrm{S} 2=0.7549 \backslash \mathrm{S} 2-1=0 . \backslash \mathrm{S} 2 \mathrm{~A}=0.750018 \backslash \mathrm{RMSD}=6.602 \mathrm{e}-09 \backslash \mathrm{RMSF}=1.901 \mathrm{e}-05$ $\backslash \mathrm{Dipole}=-0.7441963,1.0912312,0.4629956 \backslash \mathrm{PG}=\mathrm{C} 01 \quad[\mathrm{X}(\mathrm{C} 7 \mathrm{H} 8 \mathrm{~N} 103)] \backslash \backslash @$

\section{(3S,5R)-C5}

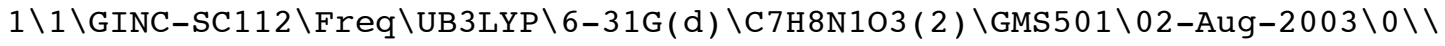
\#N GEOM=ALLCHECK GUESS=READ SCRF=CHECK TEST GENCHK UB3LYP/6-31G(D) FRE $\mathrm{Q} \backslash \backslash 3 \mathrm{~S}, 5 \mathrm{R}$ carbapenam-3-carboxylate (c5 radical) b3lyp/6-31G* carc-g $\backslash \backslash 0$, $2 \backslash \mathrm{C},-0.4869582565,2.0192614017,0.5091432797 \backslash \mathrm{C},-1.4355221917,1.00914380$ $51,-0.0724380074 \backslash \mathrm{N},-0.6635153972,0.0278062183,-0.756077659 \backslash \mathrm{C}, 0.7599706$ $289,0.3346285396,-0.8120059893 \backslash \mathrm{C}, 0.7499356254,1.8514054921,-0.41821161$ $64 \backslash \mathrm{C},-2.3647934243,-0.0674676625,0.4895555937 \backslash \mathrm{C},-1.4271689583,-1.05725$ $76006,-0.2558683318 \backslash 0,-1.3103010019,-2.2460305007,-0.3899839728 \backslash \mathrm{C}, 1.53$ $48214131,-0.4641592124,0.2335218483 \backslash \mathrm{H}, 1.196078267,0.1663047935,-1.7993$ $16761 \backslash \mathrm{H}, 1.6882581317,2.1608591161,0.0510606885 \backslash \mathrm{H}, 0.620137918,2.4418518$ $803,-1.3306334038 \backslash \mathrm{H},-0.2254062459,1.7663538493,1.5489153633 \backslash \mathrm{H},-0.86197$ $31134,3.0482888137,0.504127164 \backslash \mathrm{H},-2.3051725978,-0.2069744827,1.5754522$ $805 \backslash \mathrm{H},-3.4129921227,-0.0572902389,0.1774559823 \backslash \mathrm{O}, 2.7845443841,-0.74995$ $97458,-0.1926271599 \backslash 0,1.1179607946,-0.7599580008,1.3304486371 \backslash \mathrm{H}, 3.2263$ $351099,-1.2197798583,0.5406016039 \backslash \backslash$ Version=DEC-AXP-OSF / 1-G03RevB.03\St ate $=2-\mathrm{A} \backslash \mathrm{HF}=-551.914396 \backslash \mathrm{S} 2=0.753163 \backslash \mathrm{S} 2-1=0 . \backslash \mathrm{S} 2 \mathrm{~A}=0.750007 \backslash \mathrm{RMSD}=5.252 \mathrm{e}-09$ 
$\backslash \mathrm{RMSF}=1.137 \mathrm{e}-05 \backslash \mathrm{Dipole}=0.728022,1.3257381,0.1018995 \backslash \mathrm{PG}=\mathrm{C} 01$

$[\mathrm{X}(\mathrm{C} 7 \mathrm{H} 8 \mathrm{~N} 103)] \backslash \backslash \mathrm{Q}$

\section{(3S,5S)-C6•}

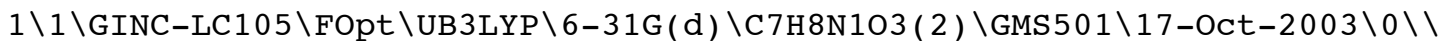
\#P B3LYP/6-31G(D) OPT=(TIGHT, READFC) FREQ TEST MAXDISK=26214400 GUESS= READ GEOM=CHECK GFINPUT IOP $(6 / 7=3) \backslash \backslash C 6$ 5S carbapenam rad b3lyp/6-31G* $\backslash 0,2 \backslash \mathrm{C}, 1.0111542411,1.0694258806,-0.63 \overline{2} 3797319 \backslash \mathrm{N}, 0.5193495779,-0.35249$ $51279,-0.5325645085 \backslash \mathrm{C},-0.5317693091,-0.4756069914,0.4778866789 \backslash \mathrm{C},-0.26$ $77328241,0.7295081507,1.4346941944 \backslash \mathrm{C}, 0.3263276972,1.8353151726,0.53214$ $74997 \backslash \mathrm{C}, 1.845311367,-0.8151585951,-0.216432265 \backslash 0,2.2321399268,-1.90071$ $7063,0.1585172003 \backslash \mathrm{C}, 2.402335248,0.5231201359,-0.4383181632 \backslash \mathrm{C},-1.931070$ $225,-0.4319879828,-0.1188554066 \backslash \mathrm{H},-0.4354794452,-1.4363810945,0.987211$ $9341 \backslash \mathrm{H}, 0.464304755,0.4059949927,2.1824747921 \backslash \mathrm{H},-1.1648939565,1.0550011$ $546,1.9699915952 \backslash \mathrm{H}, 1.0225910408,2.4808051657,1.0757469647 \backslash \mathrm{H},-0.4681338$ $655,2.466458014,0.1240294875 \backslash \mathrm{H}, 0.8080471127,1.5314435168,-1.6036498406$ $\backslash \mathrm{H}, 3.395157547,0.9545468651,-0.3875824535 \backslash \mathrm{O},-2.8622994974,-1.103697660$ $6,0.2605393902 \backslash 0,-2.0460295427,0.4970117344,-1.1007640772 \backslash \mathrm{H},-2.9748644$ $982,0.4611265721,-1.3990678641 \backslash \backslash$ Version=x86-Linux-G03RevB.03 $\backslash$ State $=2-\mathrm{A}$ $\backslash \mathrm{HF}=-551.9129873 \backslash \mathrm{S} 2=0.756602 \backslash \mathrm{S} 2-1=0 . \backslash \mathrm{S} 2 \mathrm{~A}=0.750029 \backslash \mathrm{RMSD}=6.037 \mathrm{e}-09 \backslash \mathrm{RMSF}=$ $6.011 e-06 \backslash \mathrm{Dipole}=-0.0806938,1.926947,-0.09127 \backslash \mathrm{PG}=\mathrm{C} 01 \quad[\mathrm{X}(\mathrm{C} 7 \mathrm{H} 8 \mathrm{~N} 103)] \backslash \backslash \mathrm{a}$

\section{$(3 S, 5 R)-C 6 \cdot$}

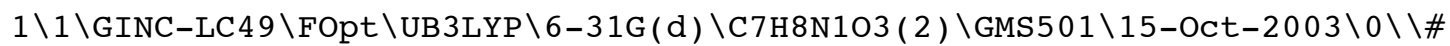
B3LYP/6-31G(D) OPT=TIGHT FREQ TEST MAXDISK=26214400\\C6_5R carbapenam $\mathrm{rad} \mathrm{b} 31 \mathrm{yp} / 6-31 \mathrm{G} * \backslash \backslash 0,2 \backslash \mathrm{C}, 1.1887290991,-1.6077809156,0.64 \overline{6} 1162429 \backslash \mathrm{C}, 1.81$ $50879824,-0.5027422383,-0.227125314 \backslash \mathrm{N}, 0.6339715869,0.1599546533,-0.869$ $0480701 \backslash \mathrm{C},-0.5864090248,-0.6572016977,-0.7948778424 \backslash \mathrm{C},-0.0692157831,-1$ $.9923228436,-0.1658631758 \backslash \mathrm{C}, 2.1511632896,0.8474579522,0.3564967178 \backslash \mathrm{C}, 0$ $.8976076764,1.3922561797,-0.1919471365 \backslash 0,0.2570703913,2.4182106617,-0$. $126690037 \backslash \mathrm{C},-1.7049857382,-0.0170280138,0.0266544634 \backslash \mathrm{H},-1.0105406509,-$ $0.8214833841,-1.7885474453 \backslash \mathrm{H},-0.8315782433,-2.486042471,0.4438426062 \backslash \mathrm{H}$ $, 0.1984991414,-2.6767838942,-0.9786253219 \backslash \mathrm{H}, 0.8993827088,-1.2048137698$ $, 1.6208653878 \backslash \mathrm{H}, 1.8590746254,-2.4575579072,0.8049062596 \backslash 0,-2.810624600$ $8,0.2260570816,-0.3938278143 \backslash 0,-1.3353151182,0.2010866817,1.3127625022$ $\backslash \mathrm{H}, 2.5322171221,-0.9070194967,-0.953198854 \backslash \mathrm{H}, 2.9606170798,1.2436257892$ $, 0.9565617391 \backslash \mathrm{H},-2.0863832784,0.6517266233,1.7428511795 \backslash \backslash$ Version $=\mathrm{x} 86-\mathrm{L}$ inux-G03RevB.03 \State $=2-A \backslash H F=-551.9099601 \backslash S 2=0.75606 \backslash S 2-1=0 . \backslash S 2 A=0.750$ $026 \backslash \mathrm{RMSD}=7.384 \mathrm{e}-09 \backslash \mathrm{RMSF}=3.747 \mathrm{e}-06 \backslash \mathrm{Dipole}=1.0411087,-1.51421,0.7332279 \backslash$ $\mathrm{PG}=\mathrm{C} 01 \quad[\mathrm{X}(\mathrm{C} 7 \mathrm{H} 8 \mathrm{~N} 103)] \backslash \backslash @$

\section{Ethanethiol}

$1 \backslash 1 \backslash G I N C-L C 132 \backslash F O p t \backslash R B 3 L Y P \backslash 6-31 G(d) \backslash C 2 H 6 S 1 \backslash G M S 501 \backslash 17-M a r-2004 \backslash 0 \backslash \backslash \# P$ B3 LYP /6-31G(D) OPT=( TIGHT, MAXCYCLE=100) MAXDISK=32768000 FREQ=NORAMAN GF

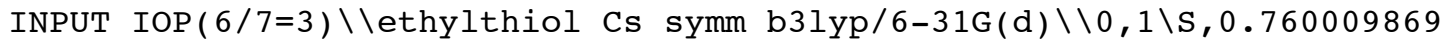
$4,-0.8487903012,0 . \backslash \mathrm{C},-0.0014702274,0.835939614,0 . \backslash \mathrm{C},-1.5230357064,0.70$ $38367679,0 . \backslash \mathrm{H}, 0.3361344411,1.3787139349,0.8876984722 \backslash \mathrm{H}, 0.3361344411,1$. $3787139349,-0.8876984722 \backslash \mathrm{H}, 2.0496000526,-0.4463835323,0 . \backslash \mathrm{H},-1.98471918$ $96,1.697680519,0 . \backslash \mathrm{H},-1.8751360265,0.1666308361,-0.8870147779 \backslash \mathrm{H},-1.8751$ $360265,0.1666308361,0.8870147779 \backslash \backslash$ Version=x86-Linux-G03RevB. $03 \backslash$ State $=1$ $-A^{\prime} \backslash \mathrm{HF}=-478.0128958 \backslash \mathrm{RMSD}=3.620 \mathrm{e}-09 \backslash \mathrm{RMSF}=1.385 \mathrm{e}-06 \backslash \mathrm{Dipole}=0.0132367,0.7$ $085161,0 . \backslash \mathrm{PG}=\mathrm{CS} \quad[\mathrm{SG}(\mathrm{C} 2 \mathrm{H} 2 \mathrm{~S} 1), \mathrm{X}(\mathrm{H} 4)] \backslash \backslash @$ 


\section{Ethanethiyl radical}

$1 \backslash 1 \backslash G I N C-L C 73 \backslash F O p t \backslash U B 3 L Y P \backslash 6-31 G(d) \backslash C 2 H 5 S 1$ ( 2$) \backslash G M S 501 \backslash 17-M a r-2004 \backslash 0 \backslash \backslash \# P$ B3LYP/6-31G(D) OPT=(TIGHT, MAXCYCLE=100) MAXDISK=34078720 FREQ=NORAMAN

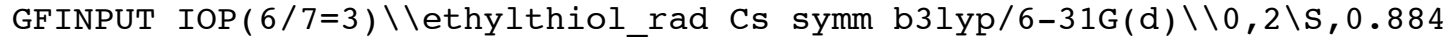
$5706217,-0.8252711103,0 . \backslash \mathrm{C},-0.0002123781,0.7661763255,0 . \backslash \mathrm{C},-1.52457268$ $6,0.6697071957,0 . \backslash \mathrm{H}, 0.3673352104,1.3228835447,0.8733903895 \backslash \mathrm{H}, 0.3673352$ $104,1.3228835447,-0.8733903895 \backslash \mathrm{H},-1.9702050541,1.6725744974,0 . \backslash \mathrm{H},-1.88$ $44424646,0.1353475253,-0.8848070522 \backslash \mathrm{H},-1.8844424646,0.1353475253,0.884$ $8070522 \backslash \backslash$ Version=x86-Linux-G03RevB.03 State $=2-\mathrm{A} " \backslash \mathrm{HF}=-477.3751414 \backslash \mathrm{S} 2=0$. $752516 \backslash S 2-1=0 . \backslash S 2 A=0.750003 \backslash R M S D=9.409 e-09 \backslash R M S F=5.527 e-06 \backslash D i p o l e=-0.36$ $09336,0.6385611,0 . \backslash \mathrm{PG}=\mathrm{CS}[\mathrm{SG}(\mathrm{C} 2 \mathrm{H} 1 \mathrm{~S} 1), \mathrm{X}(\mathrm{H} 4)] \backslash \backslash @$ 
TABLE S2: G3(MP2)-RAD//B3-LYP/6-31G(d) Total Energies (Hartrees) at $0 \mathrm{~K}$

\begin{tabular}{|c|c|}
\hline Species & \\
\hline$(3 S, 5 S)-\mathbf{1}$ & -551.79337 \\
\hline$(3 S, 5 R)-\mathbf{1}$ & -551.79255 \\
\hline$(5 R)-2$ & -550.58136 \\
\hline$(3 S, 5 S)-\mathbf{C 1} \bullet$ & -551.13771 \\
\hline$(3 S, 5 R)-\mathbf{C 1} \bullet$ & -551.13255 \\
\hline$(3 S, 5 S)-\mathbf{C 2 \bullet}$ & -551.13689 \\
\hline$(3 S, 5 R)-\mathbf{C} 2 \bullet$ & -551.13432 \\
\hline$(5 S)-\mathbf{C 3} \bullet$ & -551.15438 \\
\hline $\mathrm{TS}:(3 S, 5 S)-\mathbf{C} \mathbf{3} \bullet \rightarrow(5 S)-\mathbf{3}$ & -551.11659 \\
\hline$(5 S)-3$ & -551.14025 \\
\hline $\mathrm{TS}:(5 S) \mathbf{- 3} \rightarrow(5 R)-\mathbf{3}$ & $-551.13082^{\mathrm{a}}$ \\
\hline$(5 R)-3$ & -551.14024 \\
\hline $\mathrm{TS}:(5 R)-\mathbf{3} \rightarrow(5 R)-\mathbf{C} \mathbf{3}$ & -551.11659 \\
\hline$(5 R)-\mathbf{C 3} \bullet$ & -551.15439 \\
\hline$(3 S, 5 S)-\mathbf{C 5 \bullet}$ & -551.13303 \\
\hline $\mathrm{TS}:(3 S, 5 S)-\mathbf{C 5} \bullet \rightarrow(3 S, 5 R)-\mathbf{C 5} \bullet$ & -551.12380 \\
\hline$(3 S, 5 R)-\mathbf{C 5} \bullet$ & -551.13585 \\
\hline
\end{tabular}


$(3 S, 5 S)-\mathbf{C 6} \bullet$

$(3 S, 5 R)-\mathbf{C 6}$

Ethanethiol

Ethanethiyl radical
$-551.13638$

$-551.13322$

$-477.40423$

$-476.76719$

${ }^{a}$ Note that an additional lower energy transition structure and minimum are present on the pathway between $(5 S)-\mathbf{3}$ and TS: $(5 S)-\mathbf{3} \rightarrow(5 R)-\mathbf{3}$. 